
allemande

46-1 | 2014

Les fondements normatifs de l'État constitutionnel moderne en Allemagne. Une approche pluridisciplinaire

\title{
Les droits publics subjectifs et la dialectique de la reconnaissance : Georg Jellinek et la construction juridique de l'État moderne
}

Olivier Jouanjan

\section{(QpenEdition \\ Journals}

Édition électronique

URL : https://journals.openedition.org/allemagne/1255

DOI : 10.4000/allemagne.1255

ISSN : 2605-7913

Éditeur

Société d'études allemandes

Édition imprimée

Date de publication : 30 juin 2014

Pagination : 51-62

ISSN : 0035-0974

Référence électronique

Olivier Jouanjan, « Les droits publics subjectifs et la dialectique de la reconnaissance: Georg Jellinek et la construction juridique de l'État moderne ", Revue d'Allemagne et des pays de langue allemande [En ligne], 46-1 | 2014, mis en ligne le 29 juillet 2019, consulté le 21 septembre 2021. URL : http:// journals.openedition.org/allemagne/1255; DOI : https://doi.org/10.4000/allemagne.1255 


\section{Les droits publics subjectifs et la dialectique de la reconnaissance: Georg Jellinek et la construction juridique de l'État moderne}

- Olivier Jouanjan*

\section{La photographie}

«Une époque dans laquelle une organisation chasse l'autre, tous les rapports publics sont conçus dans une permanente indécision, dans laquelle ce qui existe est à tout instant menacé d'un bouleversement, une telle époque n'est guère favorable à la science du droit, c'est une époque de la politique et non du droit. » Ainsi s'exprime Carl Friedrich Gerber ${ }^{(1)}$ en 1852 pour signifier que les temps de l'instabilité politique sont passés et que l'époque d'une «véritable» science du droit public est venue. L'époque de la science allemande du "droit de l'État » - le Staatsrecht - naît ainsi du désastre de la Révolution de 1848-1849. Le temps de la réaction monarchique est aussi le temps d'une "véritable " science juridique de l'État allemand. Il s'agit d'une science juridique de l'État fondée sur l'étude des États monarchiques allemands restaurés après l'effondrement du mouvement révolutionnaire en 1849, la science donc d'un droit commun de ces États. L'effort trouvera son accomplissement en 1865 avec la publication par Gerber de ses Grundzüge des deutschen Staatsrechts ${ }^{(2)}$ dans lesquels la personnalité juridique est enfin reconnue à l'État: reconnaître l'État comme une personne, c'est déplacer l'imputation de la volonté publique du monarque à l'entité abstraite État. Mais cette volonté de la personne État est une volonté spécifique, qui se démarque de la volonté des personnes privées par sa modalité propre, la domination (Herrschaft). En voulant, l'État peut obliger ses sujets, ses Untertanen sans qu'il ait besoin de leur consentement: ainsi, par le concept de domination se trouve marqué le critère de distinction et de démarcation entre le droit privé et le droit public.

* Professeur de droit public, Universités Panthéon-Assas (Paris 2) et Albert-Ludwig (Fribourg-en-Brisgau).

1 Ueber öffentliche Rechte (1852), rééd. Tübingen, Mohr Siebeck, 1913, p. 11.

$21^{\text {re }}$ édition, Leipzig, Tauchnitz, 1865. 
Je ne veux pas revenir ici sur les difficultés qui expliquent pourquoi Gerber, en 1852 encore, refusait l'idée même de personnalité de l'État ${ }^{(3)}$ : dans l'histoire des doctrines juridiques modernes, celle-ci avait été proposée par le camp libéral en 1837 par Eduard Wilhelm Albrecht, un libéral germaniste et, qui plus est, l'un des « Sept de Göttingen " ${ }^{(4)}$. L'État-personne devient nécessairement le centre d'imputation de la souveraineté et le monarque se trouve réduit à n'être plus le détenteur originaire et personnel de cette souveraineté, seulement un «organe » de l'État souverain. La solution de Gerber, en 1865, son compromis historique, sera de faire du monarque l'organe central de l'État, c'est-à-dire celui qui concentre en lui l'expression de la volonté de la personne État. Ainsi peuvent se combiner le constitutionnalisme moderne et le «principe monarchique».

L'œuvre de Paul Laband, le grand juriste allemand de Strasbourg, consistera - pour simplifier à l'extrême - à adapter la construction juridique de l'État proposée par Gerber aux conditions nouvelles imposées par l'unification, par la fondation en deux temps (1866-1871) de l'Empire allemand ${ }^{(5)}$. Comment penser juridiquement la fédération de ces États monarchiques et comment donc penser la souveraineté dans l'Empire, sans la diluer? La réponse au problème de la fédéralisation du principe monarchique tient dans l'idée d'une république de monarchies dans laquelle l'organe souverain est l'assemblée des monarques, c'est-à-dire non pas l'empereur, qui n'a pas la qualité de monarque, mais le Bundesrat, l'organe de représentation des exécutifs monarchiques auprès de la fédération ${ }^{(6)}$.

Ces juristes allemands de la seconde moitié du $\mathrm{XIX}^{\mathrm{e}}$ siècle ont inventé, grâce au concept de personnalité juridique, la figure juridique moderne de l'État. Ils l'ont inventée non pas au sens de l'« inventeur » d'une grotte mais bien au sens de l'« inventeur » du concours Lépine: avec des matériaux recyclés, ils ont construit une nouvelle machine juridique, l'État. Je souligne rapidement ce point, car on voit traditionnellement, dans l'école "Gerber-Laband », la grande école du positivisme allemand de droit public: il s'agissait en effet de dépolitiser la science juridique de l'État pour établir tout le droit public sur la base de concepts " purement » juridiques. Mais la source de cette pureté n'est pas dans la loi, dans la loi constitutionnelle, elle n'est dans aucun texte puisqu'aucun texte ne définissait l'État, la souveraineté, puisqu'aucun texte ne contenait même le mot "Herrschaft ", puisqu'aucun des concepts fondamentaux du droit public n'était déterminé par ce qu'on appelle le droit positif.

Cette remarque ne vaut d'ailleurs pas seulement pour le droit public allemand de l'époque: les «textes » du droit positif (civil ou public), les «lois » définissent rarement ou très insuffisamment des notions qui pourtant fonctionnent comme les concepts fondamentaux du droit, par exemple le «droit subjectif ». C’est en puisant dans les trésors de leur imagination que les juristes construisent les machines fictives grâce

3 Olivier Jouanjan, Une histoire de la pensée juridique en Allemagne, Paris, PUF, 2005, p. 187 sq.

4 Eduard Albrecht, "Rezension über Maurenbrechers Grundsätze des heutigen deutschen Staatsrechts ", Göttingische Gelehrte Anzeigen, 1837.

5 Paul Laband, Das Staatsrecht des Deutschen Reiches, 4 vol., Tübingen, Mohr Siebeck, 1911-1914 ( $5^{\mathrm{e}}$ éd. ; la première édition commence en 1876).

6 O. Jouanjan, Une histoire de la pensée juridique (note 3), p. 255 sq. 
auxquelles ils peuvent faire fonctionner - plus ou moins bien - les « textes ", le droit. "Construction » était d'ailleurs le nom commun par lequel ils désignaient l'essentiel de leur activité: à la fin du XIX ${ }^{\mathrm{e}}$ siècle, Jhering notait que, de la même façon que les dames ne sortent jamais sans leur crinoline, les juristes ne sortent jamais sans leur « construction ${ }^{(7)}$.

Le seul problème que rencontre le juriste dans ce travail d'imagination de ses objets fondamentaux vient de lui-même: lorsqu'il s'illusionne sur la qualité de ces objets, qu'il n'y voit pas des images, mais des substances et que, ce faisant, il donne sa méthodologie pour une ontologie. Or les juristes sont platoniciens par intérêt: seules les idées pures et plus réelles que le "réel " peuvent assurer et assumer ce que Gerber appelle la "garantie de la vérité conceptuelle » et masquer ainsi tout ce qu'il y a d'intention, d'intervention, d'imagination dans leur travail.

Pour des raisons qu'on ne peut évoquer ici, la doctrine du droit public weimarien s'est, pour une bonne partie d'entre elle, insurgée contre ce " positivisme » ou, plus exactement, ce " conceptualisme » de la doctrine impériale classique. La " querelle» weimarienne « des méthodes et des approches » devait radicaliser les oppositions et simplifier le paysage doctrinal pour désigner l'ennemi commun " positiviste ». En même temps elle fixa l'image de cette doctrine pour l'historiographie à venir et c'est ainsi que, sur la photo intitulée «Le groupe des affreux positivistes du droit public de l'Empire allemand ", au premier plan, on mit non seulement Gerber et Laband, mais on ajouta Georg Jellinek (1851-1911) qui apparaissait comme la culmination et l'accomplissement mêmes de l'École. Pourtant cette photo " historique ", c'était un peu comme les photos retouchées au bon vieux temps de l'URSS, un montage dont plusieurs versions pouvaient circuler selon les époques: on mettait toujours les trois chefs et, à l'arrière-plan, on effaçait ou rajoutait des personnages selon les besoins politiques du moment. C'est ainsi que sur certaines versions on voit distinctement le jeune Kelsen, qui venait de publier en 1911 son premier grand livre, les Hauptprobleme der Staatsrechtslehre, dans lequel pourtant il démolissait à grands coups de pioche vigoureux toute cette doctrine classique, mais qu'on avait besoin, dans l'« autre camp », de déguiser en « exécuteur testamentaire de l'école Gerber-Laband ». En même temps, le jeune Kelsen, de son côté, avait tant besoin d'affirmer son originalité et, pour ce faire, de démolir sans précaution certains monuments de valeur particulière, que même ceux qui aujourd'hui encore reconstituent l'histoire de la construction de la grande pyramide kelsénienne ne peuvent ou ne veulent pas y voir les quelques pierres - peut-être peu nombreuses, mais des grosses et des porteuses - qui manifestement provenaient de la carrière qu'avait déjà creusée Jellinek dans la science juridique classique. De sorte qu'à un moment donné, du fait d'une alliance objective de Kelsen avec ses ennemis, tout conspira à rabattre la figure de Jellinek dans l'« École ", à effacer ce qui restait de traces de montages sur la photo tandis que Kelsen s'acharnait à se faire disparaître du portrait de groupe avec dame en crinoline.

Mon propos consistera donc à dire à la fois pourquoi il vaudrait mieux effacer Jellinek de la photo et pourquoi on pourrait sans incongruité se représenter un tableau

7 «Über die civilistische Konstruktion », in: JHERING, Scherz und Ernst in der Jurisprudenz, Leipzig, Breitkopf \& Härtel, 1884, p. 7. 
représentant ensemble Jellinek et Kelsen sans, bien sûr, les identifier, ce qui serait absurde. Mais on verrait alors, peut-être, quelque chose qui ressemblerait à un nain sur des épaules de géant même s’il est évidemment inconvenant de figurer Kelsen sous les traits d'un nain. On rappellera seulement que le nain voit plus loin. En revanche, il n'est pas absolument exagéré de redonner à Jellinek sa stature de géant, car, au fond, Alexander Somek n'a peut-être pas tout à fait tort d'affirmer, avec toute la radicalité qui le caractérise, qu'on peut voir en Jellinek le véritable « fondateur de la science européenne du droit public ${ }^{(8)}$.

\section{Le problème}

«Fondateur de la science européenne du droit public », Jellinek le serait en ce sens que, mettant clairement en évidence le problème que l'approche "Gerber-Laband » ne parvient pas à résoudre, en montrant pourquoi elle ne peut le résoudre, il formule une proposition de théorie juridique et constitutionnelle qui, à la fois, paraît autoriser la science du droit public à sortir de l'aporie où elle s'est enfermée, et critique les présupposés fondamentaux de cette école dont, de ce fait, il sort radicalement ${ }^{(9)}$. Cette sortie, qui passe par une position méthodologique et théorique quant au droit, doit obliger à poser à nouveaux frais la quaestio diabolica du rapport entre théorie juridique et philosophie politique qui constitue, à mon sens, l'intrigue la plus difficile dans l'interprétation de Jellinek comme, d'ailleurs, dans l'interprétation de Kelsen : il n'est guère difficile de voir comment, chez Gerber et Laband, la « dépolitisation » de la science du droit public masque une position politique banale, conservatrice, comment il s'agit de donner au système monarchique allemand la "garantie de la vérité conceptuelle ». Kelsen lui-même dans ses Hauptprobleme der Staatsrechtslehre de 1911, dans son Allgemeine Staatslehre de 1925 ou encore dans la première édition de la Reine Rechtslehre de 1934 n'a guère de mal à démasquer les intentions certes tacites, mais partout affleurantes qui président à la science de l'École, par les moyens, somme toute assez rudimentaires, de la «critique de l'idéologie». La « défétichisation » des concepts juridiques ${ }^{(10)}$ à laquelle se livre Kelsen, son approche donc en termes de «théorie de la connaissance » rendent chez lui la question de cette articulation entre «science du droit » et "théorie politique » autrement plus subtile et complexe ${ }^{(11)}$. Mais la même chose vaut pour Jellinek et ce, pour la même raison de fond qui réside dans cette entreprise de défétichisation des concepts juridiques.

Le problème du droit public impérial au moment où, en 1892, Jellinek publie son System der subjektiven öffentlichen Rechte ${ }^{(12)}$ tient à l'incapacité théorique dans laquelle

8 «The Indelible Science of Law ", International Journal of Constitutional Law (I.Con), vol. 7, n 3 (2009), p. 441.

9 O. Jounnjan, Une histoire de la pensée juridique (note 3), p. 283 sq. et « Jellinek ou le juriste philosophe », préface à Georg JellineK, L'État moderne et son droit, t. 1, Paris, Éd. Panthéon-Assas, 2005 (rééd.).

10 J'emprunte l'expression à Philippe Raynaud qui l'emploie à propos de l'entreprise wébérienne: Max Weber et les dilemmes de la raison moderne, Paris, PUF (coll. «Quadrige »), 1996, p. 37 sq.

11 Carlos-Miguel Herrera, Théorie juridique et politique chez Hans Kelsen, Paris, Kimé, 1997; Olivier JouAnjan (dir.), Hans Kelsen, forme du droit et politique de l'autonomie, Paris, PUF, 2010.

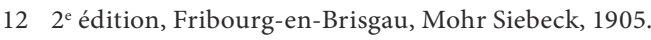


l'École se trouve de penser et donc « construire » la catégorie des droits publics subjectifs. Nous devons comprendre à la fois pourquoi cette catégorie est inconstructible et pourquoi il y a là un problème. On commencera par la seconde question.

Il ne s'agit pas d'un problème en soi - car on peut bien imaginer une construction " objectiviste ", sans droits subjectifs, du droit public et c'est une ligne française du droit public (Léon Duguit au premier chef $^{(13)}$ ) - mais d'un problème dans le contexte de la science allemande du droit. Il suffit de rappeler que, dans le champ du droit privé allemand de l'époque, le droit est pensé d'abord en termes de droits subjectifs, parce que la "pandectistique " ${ }^{(14)}$ allemande de la seconde moitié du XIX ${ }^{\mathrm{e}}$ siècle reste tributaire de l'école historique, de ses grands prédécesseurs que furent Savigny et son principal disciple, Puchta. Ce dernier fut le maître de Gerber. Il transmet à ce dernier non seulement une vision « subjectiviste » du droit - la catégorie du droit subjectif est première - mais aussi un certain concept précis du droit subjectif: c'est un pouvoir, une puissance de la volonté, Willensmacht, puissance initiale et fondatrice codée $e^{(15)}$, canalisée par le droit objectif, c'est-à-dire le système des règles de fond, de forme et de procédure. Au commencement est la volonté. Le droit est donc codage, canalisation, restriction de la libre volonté initiale des hommes; il est, comme le dit Puchta, et Gerber à sa suite, un "système des possibilités de la volonté ». Cela marche pour construire le système du droit privé, car l'égalité postulée - fictive - des volontés en concours permet de comprendre ce « code » comme équilibrage de volontés concurrentes et de même valeur: la liberté d'une volonté consiste à pouvoir faire ce qui ne nuit pas à une autre volonté et la loi, la règle objective, détermine cette limite qui se conçoit comme équilibre, institue un code rationnellement évaluable des possibilités de la volonté. Mais si le droit public se distingue du droit privé, trouve son autonomie disciplinaire dans l'idée de domination, alors le code des volontés dans le droit public ne peut pas être un code équilibrateur - ou équilibriste, c'est selon. La volonté qui seule peut dominer, c'est celle de la personne État. Comment reconnaître de la puissance, du pouvoir juridique à la volonté qui lui fait face? Comment admettre l'idée d'une volonté juridiquement efficace face à la puissance de la volonté de puissance? Face à une volonté conçue comme souveraine? C’est analogiquement le même problème que celui qui oppose, au plan théologique, partisans de la prédestination et partisans du libre arbitre: comment penser que ma volonté, qui présiderait à mes actions bonnes, puisse conditionner, déterminer et limiter la volonté souveraine de Dieu? L'école Gerber-Laband me semble être prédestinatrice: le statut juridique du sujet de l'État, de l'Untertan, est complètement et parfaitement (vollkommen) défini, juridiquement, comme objet (Gegenstand) de la puissance étatique, de la Staatsgewalt. À défaut de quoi c'est, tout à la fois, les idées de Herrschaft et de souveraineté qui seraient conceptuellement détruites.

13 Duguit (1856-1928) fut le chef de file de l'école dite "du service public ». Voir: Olivier JouANJAN, «Duguit et les Allemands », in: Fabrice Melleray (dir.), Autour de Léon Duguit, Bruxelles, Bruylant, 2010.

14 C'est-à-dire la science des Pandectes, le droit romain consigné dans le Digeste de Justinien formant, jusqu'à l'entrée en vigueur du Code civil allemand en 1900, le droit civil commun allemand.

15 Sur l'idée de « codage » je renvoie à Walter PAuly, Der Methodenwandel im deutschen Spätkonstitutionalismus, Tübingen, Mohr Siebeck, 1993, p. 107 sq. 
Mais alors, dit Jellinek, comment comprendre le « rapport de droit public »? Un " rapport de droit » est compris comme la situation, plus ou moins complexe, dans laquelle des prétentions légitimes, appuyées sur des droits subjectifs, se font face et sont, le cas échéant, présentées au juge afin que, par application du code équilibrateur qu'est le droit objectif, celui-ci tranche entre ces prétentions. Mais il n'y aurait aucun équilibre pensable dans le sous-système du droit public, aucun " rapport de droit " stricto sensu, et donc aucune garantie judiciaire véritable pour l'individu "face » à l'État. Le «face-à-face » entre l'État et l'individu ne serait juridiquement ni pensable ni possible. Dans le droit public il n'y aurait qu'une seule personne, isolée, celle de l'État, en face de quoi il n'y aurait que des objets, les Untertanen. Or, un objet juridique n'a aucune prétention à faire valoir face à un Sujet de droit, l'État, et l'idée même d'un rapport de droit public n'est pas imaginable. Mais si le « rapport de droit » est une catégorie fondamentale du droit, alors c'est le droit public lui-même qui n'est ni imaginable ni possible. Or, le rapport de droit et le droit subjectif sont, dans cette culture juridique allemande, les catégories fondamentales à travers lesquelles on peut seulement penser le droit. Donc, le problème de la doctrine Gerber-Laband constitue, dans la construction acceptée du droit allemand, une aporie: la possibilité d'un droit public est sapée à la base par la doctrine même qui prétend fonder la «science véritable » de ce droit.

\section{L'instrumentarium}

Comment donc imaginer le droit public? Telle est la question. L'imagination n'est pas un concept de Jellinek. Mais peut-on penser sans métaphores ${ }^{(16)}$ ? La question vaut pour la pensée juridique. La métaphore et le concept sont des optiques par lesquelles nous pouvons seulement considérer les choses qui, grâce à elles, deviennent des objets pour nous. Il me semble que, même si ce thème de l'imagination ne se trouve pas chez Jellinek, il en est proche en raison de sa théorie de la connaissance.

Jellinek voulait être philosophe et non juriste. Sur son chemin de formation il a croisé le renouveau du kantisme. Un ami fidèle, jusqu'à la fin de ses jours, fut Wilhelm Windelband, l'une des figures majeures du «néo-kantisme ». De ce fait, Jellinek se pose des problèmes de «théorie de la connaissance » qui n’étaient certainement pas ceux de la génération antérieure. Il s'inscrit dans le débat sur la spécificité des sciences de l'esprit (Dilthey) ou des sciences de la culture (Rickert). La question de la conceptualisation dans ces sciences est alors décisive. Jellinek se trouvera au centre du monde de l'esprit allemand "fin de siècle ", à Heidelberg, où il fréquente assidûment Max Weber et son cercle. Mais il a formulé sa position méthodologique dès avant sa rencontre avec les grands fondateurs de la sociologie allemande, dans la "partie générale » de son System der subjektiven öffentlichen Rechte. La première édition de l'Allgemeine Staatslehre (L'État moderne et son droit) date de 1900 et l'on y trouve, avant la parution du célèbre essai de Max Weber sur l'objectivité en sciences sociales, une théorie des "types », le type étant, selon lui, la forme spécifique de conceptualisation dans les sciences sociales. On y trouve une «théorie sociale de l'État», bien avant la grande sociologie wébérienne de l'État. La question de l'articulation entre la théorie sociale et la théorie juridique de l'État est difficile. Ces « deux faces » de l'État sont souvent présentées comme étant 
posées l'une à côté de l'autre sans connexions internes. Cette interprétation traditionnelle ne voit pas ce qu'est, chez Jellinek, un " concept » juridique, un «type ».

Si la connaissance débute dans la sensibilité, elle ne s'épuise pas en elle: il y faut le concept; la sensibilité est " réceptrice ", la conceptualisation est "spontanée » et passe par une prestation propre du sujet de la connaissance. Jellinek est néo-kantien: l'on ne peut connaître la « chose en soi » mais seulement le phénomène pour nous, l'objet construit par nous et par concepts. Il n'y a pas de connaissance possible de l'État «en soi ». L'État n'est rien d'autre qu'un système particulier de relations interindividuelles que, par la connaissance, on isole et caractérise par concept. Il faut souligner deux aspects fondamentaux pour comprendre ce qu'est, pour Jellinek, un concept opératoire dans la science juridique.

D'une part, les concepts juridiques doivent reposer sur une base empirique: la connaissance commence dans la sensibilité. D’où la nécessité qu'il existe un ensemble de relations interindividuelles synthétisées sous le concept sociologique d'État, sans quoi il ne peut y avoir de théorie juridique possible de l'État. Le concept de l'État n'est qu'une typification d'un système particulier de relations sociales, donc non pas au sens propre une fiction, mais une abstraction. D'autre part, les concepts juridiques ne sauraient dire l'en soi, la substance des objets juridiques: ils ne sont que des synthèses de phénomènes permettant de faire fonctionner le discours d'une science particulière qui a pour but non pas de décrire le monde mais de systématiser un ensemble de normes humaines. La question scientifique n'est pas « qu'est-ce que? » - la propriété, le contrat, l'État - mais «comment dois-je penser? " - la propriété, le contrat, l'État - en fonction du point de vue adopté, ici du point de vue d'un ordre juridique. Pour le droit public, la question est donc: "Comment dois-je penser juridiquement l'État? " ${ }^{(17)}$. Comment dois-je donc construire mon objet juridique « afin que l'ensemble des normes qui s'y rapportent puissent être ramenées à une unité sans contradiction " ${ }^{(18)}$ ?

Ces deux principes sont ramassés dans le passage suivant: « Les concepts juridiques n'ont aucune essence pour objet, le monde juridique est un pur monde de la pensée qui est au monde réel ce que la sensation esthétique est à la connaissance théorique. Mais il est un monde des abstractions et non des fictions. Il y a à la base de l'abstraction des processus réels dans le monde des événements extérieurs et intérieurs quand la fiction, en revanche, met à la place du fait naturel un fait imaginé et le met à égalité avec ce dernier. L'abstraction repose sur l'événement, la fiction, sur l'invention " ${ }^{(19)}$. Les concepts juridiques sont donc des synthèses du sujet de la connaissance qui doivent avoir une base empirique. Cette base n'est faite ni de "substances " ni de " qualités ", mais de "processus psychiques de masse extrêmement embrouillés et de relations des individus entre eux » qui, par synthèse, conduisent à la " représentation de droits subjectifs et d'institutions juridiques objectives " ${ }^{(20)}$. Jellinek accomplit un pas décisif dans la voie de la « défétichisation » des concepts juridiques et dans la distinction entre « concepts substantiels » et « concepts fonctionnels».

17 G. JeLlinek, System (note 12), p. 20.

18 Ibid., p. 23.

19 Ibid., p. 23-24.

20 Ibid., p. 22. 


\section{La reconstruction}

Cette critique méthodologique explique pourquoi Jellinek prend radicalement ses distances à l'égard de la construction « gerbero-labandienne » du droit public: ceux-ci pensent l'État comme substance (même «fictive ») et arrivent ainsi à l'aporie indiquée plus haut. L'État serait comme une personne dont la volonté se forme en son for intérieur puis s'exprime par la déclaration de cette volonté (l'acte juridique, la loi, le règlement). Cette volonté étant "souveraine " (la souveraineté serait une qualité substantielle de l'État), aucune volonté ne peut lui résister et donc aucun droit subjectif de l'individu n'est en mesure, juridiquement, de limiter cette volonté de l'État: comment un individu pourrait-il avoir un droit - exigible, opposable - sur l'exercice d'une puissance souveraine? Mais alors l'État dispose de ses sujets, juridiquement, comme s'il s'agissait d'objets de sa volonté. Or, si le droit est mesure et limite, comment penser un droit public, dans un modèle qui reste fondamentalement « subjectiviste » dès lors qu'aucun droit n'est opposable à l'État?

Jellinek déplace la catégorie dans laquelle celui-ci doit être pensé: non pas dans la catégorie de la substance, mais, parce qu'il n'est que la typification synthétisante et abstractrice que nous faisons, du point de vue juridique, d'un ensemble complexe de relations interindividuelles, dans la catégorie de la relation. Il faut désubstantialiser l'État et la personne en général, penser cette dernière comme un système de relations et donc liée à d'autres personnes co-relatives. Par ailleurs, on ne peut penser juridiquement la personne comme précédant l'ordre du droit, mais seulement comme le concept premier - si l'on adopte le point de vue subjectiviste - d'un ordre juridique qui codifie un système complexe de relations. Dès lors, la représentation que se fait l'École d'un État-personne isolé au sein du droit public est absurde, " ein Unding » dit Jellinek. On est donc amené à reconstruire juridiquement l'État par une contrainte qui n'est pas externe mais interne au discours de la science du droit, une contrainte imposée par une double logique, celle (néo-kantienne) de la connaissance scientifique du droit et celle (qu'impose le contexte subjectiviste de la pensée juridique allemande que ne remet pas en cause Jellinek ${ }^{(21)}$ ) de la construction des sujets du droit public.

La personne juridique est " relation d'un sujet à d'autres sujets et à l'ordre juridique ", une relation « abstraite " ${ }^{(22)}$. Le concept de l'État comme personne interdit donc qu'on le conçoive comme substance séparée mais bien comme fondamentalement ouverture et liaison à autrui: l'altérité, l'autre sujet, est intégrée au concept même de l'État-relation. D’ailleurs, si la personnalité vient non de la nature mais de l'ordre juridique et si l'État est le maître de l'ordre juridique, alors c'est par et dans l'État que l'individu et les groupes privés reçoivent leur personnalité. Celle-ci, dit Jellinek, a son fondement dans le droit de l'État. Jellinek n'est pas loin de penser que, juridiquement, l'État n'« est » pas autre chose qu'un ordre juridique ${ }^{(23)}$. En tout cas, il n'est pas juridiquement autrement pensable que comme un système de rapports de droit.

Cela entraîne une première conséquence sur le plan de la construction juridique: l'État est personne mais, plus précisément, personne corporative, notion décisive pour

21 Différence capitale avec l'« objectivisme » kelsénien.

22 G. Jellinek, System (note 12), p. 34.

23 Il s'agit d'une thèse célèbre et capitale du kelsénisme. 
comprendre plusieurs aspects essentiels du travail dogmatique de Jellinek sur le droit public (notamment sa théorie du peuple comme " organe d'État »). Mais cela veut aussi dire: les individus qui forment le peuple (la doctrine classique n'arrive pas à penser la question de l'étranger!) sont, conceptuellement, juridiquement incorporés à l'État, sous le rapport du droit public (pas sous le rapport du droit privé). Ils le sont, parce que leur personnalité - leur qualité juridique abstraite - est précisément de droit public ${ }^{(24)}$ : c'est dans cette relation à l'État qu'ils acquièrent leur personnalité. C'est une incorporation seulement de droit public (ce qui doit permettre de penser la représentation) et une incorporation sous le seul signe de la relation, en aucun cas une incorporation substantielle, de type organiciste: Jellinek est un «individualiste méthodologique » et donc aussi un « individualiste politique » en lutte contre tous les « organicismes » qui hantent la science juridique allemande. S’il prend à Gierke, le grand représentant de l'organicisme juridique de son époque, la notion de corporation, c'est sans doute parce qu'il trouve chez celui-ci l'idée que la corporation permet de penser les relations juridiques entre les dirigeants de la corporation et les simples membres de la corporation comme de « véritables rapports de droit " ${ }^{(25)}$ et c'est précisément ce qu'il s'agit de construire pour le droit public: la relation gouvernants-gouvernés comme un "véritable rapport de droit ». Mais il s'agit en même temps de désubstantialiser l'idée de corporation: elle n'est qu'une catégorie abstraite.

Le premier pas consiste à penser le véritable rapport de droit entre l'État et ceux qui ne lui sont pas extérieurs, mais relationnellement incorporés, les membres de l'État qui doivent donc être conçus, par contrainte logique, comme des personnes et donc des titulaires de droits subjectifs dans leur relation à l'État. Pour comprendre la très célèbre théorie jellinékienne de l'" autolimitation de l'État ", il ne faut jamais perdre de vue ce déplacement fondamental dans le concept de personnalité de l'État: il serait vraiment absurde de penser qu'une personne souveraine, substantielle, se limiterait par sa seule et propre volonté puisque ce qu'elle veut aujourd'hui ne saurait lier son vouloir demain. Duguit et Kelsen ont fait ce reproche à Jellinek, mais celui-ci est lui-même absurde car il recourt ce faisant à un concept de personne qui n'est précisément pas celui de Jellinek. Au-delà, c'est parce que l'individu doit être pensé lui aussi juridiquement comme personne, au sens d'un système de relations juridiques, que la catégorie des droits publics subjectifs devient pensable et, si l'on reste fidèle au principe subjectiviste du droit, nécessaire pour reconstruire tout le système du droit public. C'est d'ailleurs pourquoi le Système des droits publics subjectifs doit être lu non pas comme un traité sur une partie du droit public, mais comme une sorte de coupe transversale de tout le système du droit public.

Pour voir ce point décisif, il convient de comprendre le sens véritable d'une autre célèbre doctrine de Jellinek, la doctrine dite des statuts. La personnalité désigne un « être " juridique: on n'a pas une personnalité, on l'est. Mais c'est un « être » purement représenté, abstrait, et qui n'est pas autre chose que relations. Jellinek y insiste constamment. Si la personne est système de relations et si la personnalité est "iuris publici », alors comprendre la personnalité juridique de l'individu, c'est comprendre les types de relations juridiques qu'il peut avoir avec l'État. Les « statuts » ne sont que les catégories

24 G. JeLlineK, System (note 12), p. 82.

25 Otto von Gierke, Die Genossenschaftstheorie und die deutsche Rechtsprechung, Berlin, Weidmann, 1887, p. 182. 
des relations de l'individu avec l'État. Il faut donc penser les relations possibles - pensables - de l'individu avec l'État. Il y en a quatre qui se croisent logiquement deux par deux. Le statut passif exprime la relation de subordination pure et simple de l'individu à l'État: l'individu est nié (pur objet de domination) et l'État affirmé (sujet de la domination). Le statut négatif est au contraire la relation dans laquelle l'individu est affirmé et l'État nié: l'individu peut juridiquement revendiquer l'abstention de l'État, c'est la sphère staatsfrei, libre d'État. Le statut positif signifie la relation dans laquelle l'État agit pour le compte de l'individu: il doit à ce dernier une prestation (matérielle ou juridique), ce qui fait naître dans le chef de l'individu une créance à l'égard de l'État. Le statut actif dit la relation dans laquelle l'individu agit pour le compte de l'État: sa volonté s'exprime dans l'intérêt de l'État, en tant qu'électeur ou que détenteur d'une fonction publique, il exerce un droit politique. Telles sont, dit Jellinek, les quatre types de relations possibles et pensables de l'individu à l'État et telles sont donc les catégories dans lesquelles on peut penser la personnalité juridique. Les statuts forment ainsi la table des catégories de la subjectivité juridique. Et le système de droits publics subjectifs, dont la doctrine des statuts est la clé (c'est grâce à elle qu'un véritable système de ces droits peut être construit), passe donc par la déduction a priori de ces catégories. Les statuts sont donc les conditions de possibilité et de pensabilité de tout système du droit public possible. Ils ne disent pas davantage et surtout ne disent d'aucune manière comment un droit public positif doit remplir les catégories, quels sont les droits qu'un État devrait reconnaître. Jellinek est positiviste, pas jusnaturaliste. La doctrine des statuts ne dit pas comment la domination étatique doit être exercée, mais comment l'on peut penser le rapport de domination comme un véritable rapport de droit, ce qui est le problème fondamental auquel se trouve confrontée toute doctrine du droit public qui fait du sujet de droit le principe de sa construction.

Enfin, Jellinek se trouve dans l'obligation de modifier le concept gerbérien du droit subjectif: la volonté de l'individu, prise comme telle, ne saurait s'opposer à la pure volonté de domination de l'État, comme on l'a dit. Mais il est impossible de penser les concepts normatifs sans l'idée de but. Les concepts du droit doivent contenir un moment téléologique, même si le contenu concret du but ne peut être déterminé $a$ priori, scientifiquement. Les concepts du droit sont des synthèses qui saisissent, dans la diversité du réel, des "unités téléologiques " (Zweckeinheiten). Ainsi l'idée de but doit-elle être contenue dans le concept juridique d'État même si scientifiquement l'on ne peut dire quels sont les buts que doit poursuivre l'État. De même pour le concept de droit subjectif: la volonté pure et simple ne peut suffire, il faut y ajouter une détermination générale de but. Les droits sont attribués « en vue de ». Et Jellinek dispose d'une définition nouvelle du droit subjectif, celle qu'en a donné Jhering dans L'esprit $d u$ droit romain. Le concept de droit subjectif doit aussi contenir un élément "matériel » complétant l'élément « formel » de la volonté. Cet élément matériel est celui de l'intérêt. Un pouvoir de volonté est conféré par le droit en vue de la défense d'un intérêt personnel du détenteur de ce pouvoir de volonté. Le droit positif définit les intérêts qui deviennent ainsi des «intérêts juridiquement protégés ».

Pour Jellinek, cette combinaison signifie que la volonté de l'individu ne doit pas être pensée comme une volonté nue, mais une volonté rattachée à des intérêts. Remplir de contenu les statuts, c'est désigner les intérêts juridiquement protégés, ceux au regard 
desquels l'État s'autolimite: le droit public subjectif n'est que l'autre face de l'autolimitation de l'État. La doctrine des droits publics subjectifs et celle de l'autolimitation se tiennent en un rapport de réciprocité logique. Et c'est pourquoi la volonté de l'individu peut être pensée comme capable juridiquement d'arrêter la volonté de l'État dès lors qu'il s'agit de protéger une sphère d'intérêts propres pour laquelle l'État s'autolimite. Le problème du droit public subjectif est ainsi « résolu » grâce à un arsenal théorique extrêmement sophistiqué.

\section{La reconnaissance}

Il n'y a qu'en pensant l'intérêt individuel que je peux construire juridiquement la limite de la domination de l'État. Cette limite (autolimitation) distingue l'individu de l'État, deux « sujets ». Il s'agit d'une distinction de sphères d'intérêts qui sont à la fois séparées et reliées: les statuts passif et négatif sont les statuts de l'exclusion réciproque des intérêts de l'État et de l'individu; les statuts positif et actif, les statuts de la coopération des intérêts. Cette distinction (exclusion/coopération) des droits paraît être la traduction juridique, chez Jellinek, de l'idée de séparation/coordination entre l'État et la société civile: il n'y a de société civile que celle que garantit la protection juridique d'intérêts distincts de ceux de l'État. Jellinek entend penser scientifiquement la possibilité de l'État libéral. Mais il est en même temps un positiviste: il ne peut justifier scientifiquement, a priori, hors du cadre formé par le droit positif d'un État, quels sont les intérêts qui doivent être juridiquement protégés et, réciproquement, quels sont les «buts de l'État». Simplement, il a établi le cadre théorique, la condition de pensabilité juridique ou encore la grammaire juridique de l'État libéral.

Si l'on considère maintenant l'autre pan monumental du travail de Jellinek l'ouvrage sur la Déclaration des droits de l'homme et du citoyen, la grande " Histoire de l'État " qu'on trouve dans L'État moderne et son droit -, on comprend pourquoi la construction de ce cadre de théorie juridique est, à son époque, une nécessité qui n'est pas dépourvue de justification politique. Tout le travail d'histoire des idées et d'histoire des institutions auquel se livre Jellinek consiste à montrer la puissance d'une dynamique profonde dans l'aventure de l'État moderne, une dynamique de la reconnaissance dont le moteur est une sorte de dialectique positive entre la reconnaissance par l'État de l'individu (de sa valeur, c'est-à-dire de ses intérêts : remplir les trois statuts qui réduisent comme dans un système de vases communicants le statut purement passif de l'individu) et, corrélativement, de reconnaissance par l'individu de l'État (de son ordre juridique, de sa légitimité).

On passera vite sur le premier aspect: il s'agit du processus historique de reconnaissance progressive et d'extension des droits de l'homme dont Jellinek, on le sait, recherche les sources doctrinales. Sur le second aspect, il faut préciser que dans la querelle qui oppose les juristes quant à la question dite du "fondement de validité du droit » - pour quelle raison une norme peut être dite valoir à titre de norme juridique? -, Jellinek prend le parti de la doctrine dite de la « reconnaissance " contre la théorie (jheringienne) de la «contrainte »: ce n'est pas la contrainte ou la menace de son emploi qui justifient l'obligatoriété du droit, mais la reconnaissance par l'opinion, la société civile du caractère obligatoire de la norme. On retrouverait ici une proximité avec les thèses de l'école historique avec laquelle il est pourtant en désaccord 
profond sur tant d'autres points et évidemment une distance considérable avec Kelsen. D'ailleurs il existe un lien profond entre cette doctrine de la reconnaissance et l'intérêt que porte Jellinek aux phénomènes de «mutations informelles » du droit, ces changements affectant l'ordre juridique sans modification des textes (de la loi, de la constitution) : il faudrait au juriste, dit-il en 1905, une théorie des "forces créatrices du droit ", des conditions sociales du changement juridique, ce qui ne se conçoit que dans le cadre d'une conception du fondement de validité du droit reposant sur la reconnaissance.

L'État a intérêt à reconnaître des droits subjectifs aux individus qui auront d'autant plus d'intérêt à reconnaître la valeur du droit positif de l'État. Pour le dire autrement, cette dialectique de la reconnaissance pose la question de la légitimité, une question à laquelle le positiviste ne peut apporter de réponse ex cathedra mais dont il peut expliciter la structure. En même temps, elle projette toute la construction théorique et scientifique du droit sur un horizon politique que, malgré toutes les précautions scientifiques prises par l'auteur, on reconnaît sans grande peine, un horizon libéral, et même d'inspiration assez tocquevillenne (Jellinek était un lecteur de Tocqueville) si l'on pense aux difficultés que posaient à Jellinek le processus de démocratisation et ses compagnons de route inévitable, les partis politiques de masse.

Il y aurait encore tant à dire sur Jellinek. Mais on s'est surtout efforcé ici, à partir de quelques questions fondamentales, de montrer ce qu'est le travail d'un grand juriste et pourquoi nous devons accorder à l'auteur du Système des droits publics subjectifs sinon de la gratitude, du moins un peu de reconnaissance.

\section{Zusammenfassung}

Wie ist das Verhältnis zwischen dem Staat und dem Einzelnen im begrifflichen System der Gerber-Laband-Schule des deutschen Staatsrechts zu begreifen? Der Beitrag nimmt die Mechanismen auseinander, die dem juristischen Konstrukt des modernen Staates zugrunde liegen und versucht dabei, den Stellenwert von Georg Jellineks Denken gegenüber dem Hans Kelsens ins rechte Licht zu rücken. Jellinek stell den subjektiven Hintergrund des deutschen Rechtsdenkens nicht in Frage. Er begreift den Staat nicht als (fiktive) Substanz, sondern als Beziehung: so löst er die Aporie auf, die durch das subjektive Rechtsmodell hervorgebracht wurde, dem es nicht gelang, ein subjektives Recht, das dem Staat entgegengesetzt werden könne, tatsächlich zu begreifen.

\section{Résumé}

Comment penser le rapport État/individu dans le système conceptuel de l'école allemande du droit public ou doctrine Gerber/Laband? L'auteur décortique les mécanismes qui sous-tendent la construction juridique de l'État moderne en s'efforçant de rétablir la place de Georg Jellinek face à la figure de Hans Kelsen. Jellinek ne remet pas en cause le contexte subjectiviste de la pensée juridique allemande. Il pense l'État non pas comme substance (fictive), mais comme relation: c'est ainsi qu'il résout l'aporie créée par le modèle subjectiviste qui ne parvient pas à penser véritablement un droit subjectif qui puisse être opposé à l'État. 